\title{
Unusual "twister"-like appearance of a sigmoid volvulus on computed tomography
}

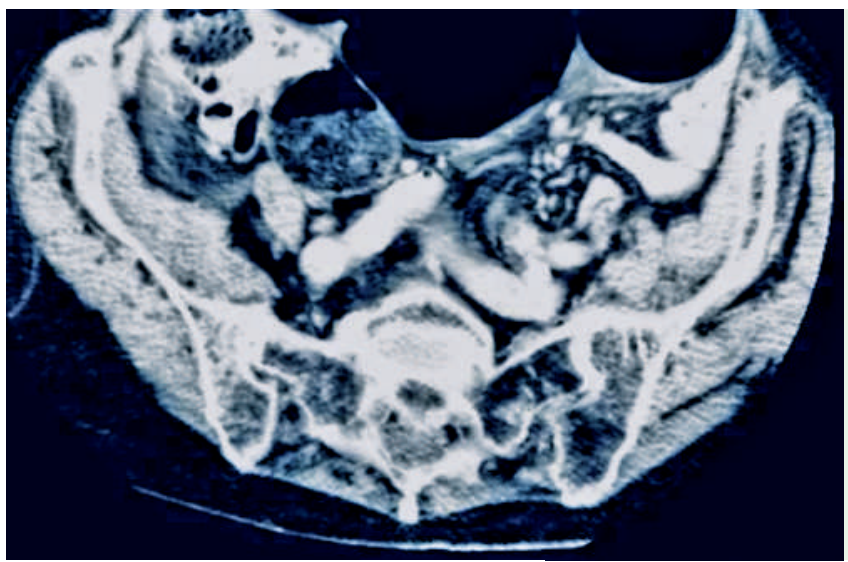

Fig. 1 Post-processing computed tomographic (CT) scan (allowing high-resolution acquisition of three-dimensional [3D] images) using a 16-slice scanner (Aquilion 16, Toshiba, Nasu, Japan) after administration of both oral and intravenous contrast media: a transverse abdominal slice of the lower abdomen.

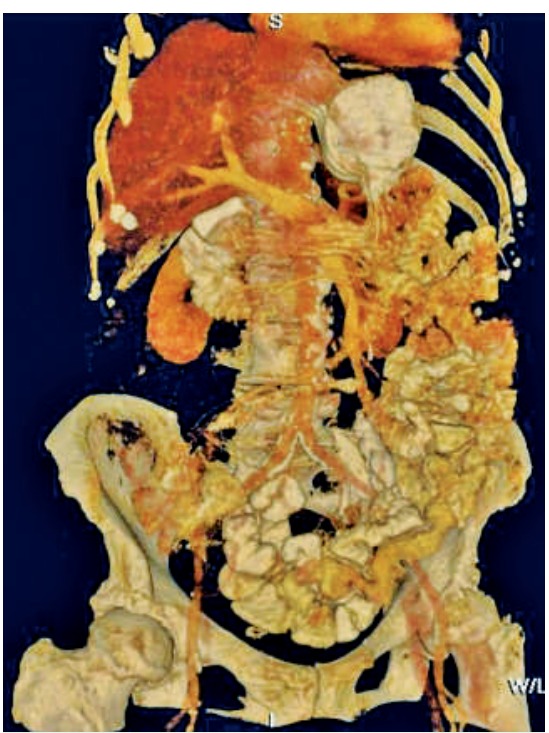

Fig. 2 A post-processing CT scan from the right frontal perspective, showing a 3D model of the twisted sigmoid colon with intact mesenteric perfusion. (The processing has substracted connective tissue but preserved all luminal structures such as vessels and intestine.)

One possible cause of ileus is the presence of a distorted intestinal segment, but this is uncommon in older patients. We report here an exception to this rule of thumb.

\section{Video 1}

A post-processing $C T$ scan (see legend for - Figure 1) after administration of oral and intravenous contrast media, moving through a portion of 286 transverse abdominal slices of the lower abdomen.
An 87-year-old woman had been admitted with a 2-day history of abdominal pain. She had a history stretching back over several years of senile dementia. Clinical and laboratory examinations led to a provisional diagnosis of ileus (the $\mathrm{C}$ reactive protein was within the normal range and the white blood cell count was $12.1 \times 10^{9} / \mathrm{L}$ ). Computed tomography revealed an impressive image of a volvulus of the sigmoid colon. The patient therefore underwent laparotomy under perioperative antibiotic cover [1], which confirmed the preoperative diagnosis. The sigmoid colon was distorted but there was no evidence of disturbance of mesenteric perfusion. This allowed untangling of the extra-long sigmoid loop, and resection was not required. In addition, the colon was decompressed by transanal placement of a decompression tube. Subsequently, the sigmoid was fixed with some stitches to the left parietal peritoneum, the inner surface of the abdominal wall.

The patient was monitored postoperatively for 3 days in the surgical intensive care unit. She developed a temporary postoperative psychotic disorder (with disorientation and deterioration in her dementia) but otherwise her postoperative course, in terms of reuptake of oral fluids and food and wound healing, was uneventful. The patient was discharged on the 10th postoperative day with no further complaints.

This short report presents extremely unusual computed tomographic images of a rare "twister"-like structure in the left lower abdomen ( $\bullet$ Fig. 1, 2; Videos 1,2) [2], which turned out to be an intestinal volvulus caused by an extra-long sigmoid colon. The images greatly facilitated the elucidation of the clinical diagnosis of an ileus by eliminating other much more likely potential diagnoses (given this patient's age), such as obstructing tumorous lesions or disturbances of mesenteric perfusion.

Endoscopy_UCTN_Code_CCL_1AD_2AJ

\section{F. Meyer ${ }^{1}$, L. A. C. Rapp², H. Lippert}

Department of Surgery, University

Hospital, Magdeburg, Germany

Department of Radiology and Nuclear

Medicine, University Hospital, Magde-

burg, Germany

\section{References}

1 Tsai MS, Lin MT, Chang KJ et al. Optimal interval from decompression to semi-elective operation in sigmoid volvulus. Hepatogastroenterology 2006; 53: 354-356

2 Hirao K, Kikawada M, Hanyu H, Iwamoto T. Sigmoid volvulus showing "a whirl sign" on CT. Intern Med 2006; 45: 331 - 332

Bibliography

DOI $10.1055 / \mathrm{s}-2007-966479$

Endoscopy 2007; 39: 295

(c) Georg Thieme Verlag KG Stuttgart · New York ISSN 0013-726X

\section{Corresponding author}

\section{F. Meyer, MD}

Department of Surgery

University Hospital

Leipziger Strasse 44

D-39120 Magdeburg

Germany

Fax: +49-391-67-15570

frank.meyer@medizin.uni-magdeburg.de

\section{Video 2}

A post-processing $C T$ scan showing a 3D model of the twisted sigmoid colon and intact mesenteric perfusion (from the left to the right frontal perspective), in which connective tissue has been substracted but all luminal structures such as vessels and intestine have been preserved. 